\title{
Turbulence measurements in fusion plasmas
}

\author{
G D Conway \\ Max-Planck-Institut für Plasmaphysik, EURATOM-Association IPP, D-85748 \\ Garching, Germany
}

\begin{abstract}
Turbulence measurements in magnetically confined toroidal plasmas have a long history and relevance due to the detrimental role of turbulence induced transport on particle, energy, impurity and momentum confinement. The turbulence - the microscopic random fluctuations in particle density, temperature, potential and magnetic field - is generally driven by radial gradients in the plasma density and temperature. The correlation between the turbulence properties and global confinement, via enhanced diffusion, convection and direct conduction, is now well documented. Theory, together with recent measurements, also indicate that nonlinear interactions within the turbulence generate large scale zonal flows and geodesic oscillations, which can feed back onto the turbulence and equilibrium profiles creating a complex interdependence. An overview of the current status and understanding of plasma turbulence measurements in the closed flux surface region of magnetic confinement fusion devices is presented, highlighting some recent developments and outstanding problems.
\end{abstract}

PACS numbers: 52.35.Ra, 52.25.Fi, 52.55.-s, 52.70.-m

Submitted to: Plasma Phys. Control. Fusion 


\section{Introduction}

One of the enduring problems in magnetic confinement of fusion plasmas is that of turbulent transport. The comprehensive theory of Hinton and Hazeltine (1976) [1] describes the cross-field diffusive transport of particles and energy due to inter-particle collisions in toroidal confinement devices (neoclassical theory). Experiments, however, show much higher transport, typically a factor of 10 for ions and upto $10^{3}$ for electrons [2]. This additional - previously termed "anomalous" - transport is generally attributed to the impact of turbulent fluctuations arising from a variety of saturated linear modes, driven primarily by the radial gradients in the plasma density and temperature.

Progress in understanding, and indeed managing, the impact of plasma turbulence has come via two sources: significant improvements in diagnostics and data interpretation, and advances in numerical turbulence simulations and modelling. The close interaction in recent years between experiment and simulation has been particularly fruitful, not only in motivating new experimental studies, but also pushing diagnostic development (a main limitation in experimental investigations) as well as revealing new insights - such as zonal flows. The aim of this overview is to summarise the recent progress in experimental turbulence measurements; highlighting the current issues, such as high- $k$ turbulence and streamers (convective transport events believed to be responsible for enhanced electron transport), zonal flows, plus the search for the linear modes behind the turbulence - particularly for transitions between the modes. Understanding of the back-reaction of the turbulence on the equilibrium fields, as well as the stabilizing effects of plasma shape, velocity and magnetic shear has also progressed. Strictly speaking, the turbulence is electromagnetic in nature, but as magnetic fluctuations are generally small the resulting turbulent transport is predominately electrostatic. However, recent experimental high $\beta$ (plasma over magnetic pressure) results are reviving the issue of electromagnetic effects.

A good starting point to the topic of fusion plasma turbulence is the review paper by Liewer [3], which gives a comprehensive status report of turbulence measurements in tokamaks and their understanding upto 1985, and the excellent survey by Wootton (1990) [4]. Much of the early measurements are still relevant, and in some areas unbettered, some two decades later. Various updates and partial reviews have appeared subsequently, including Sheffield (1994) [5], Hugill (2000) [6] and Burrell (2006) [7]. The best review of diagnostic techniques for turbulence measurements is still Bretz's comprehensive 1997 paper [8] which covers all of the relevant techniques and applications. One notable new diagnostic technique is Doppler reflectometry, cf. [9].

Although the emphasis here will be on turbulence in the confined region of closed magnetic flux surfaces, there has also been much work on the surrounding scrape-offlayer (SOL) where the magnetic field lines terminate on a limiter or divertor surfaces. Recent reviews here include Hidalgo (1995) [10] and Carreras (2005) [11]. Zweben (2007) [12] also gives a survey of SOL turbulence measurement techniques. In the broader context there are also review papers on confinement in toroidal devices [13, 14] and 


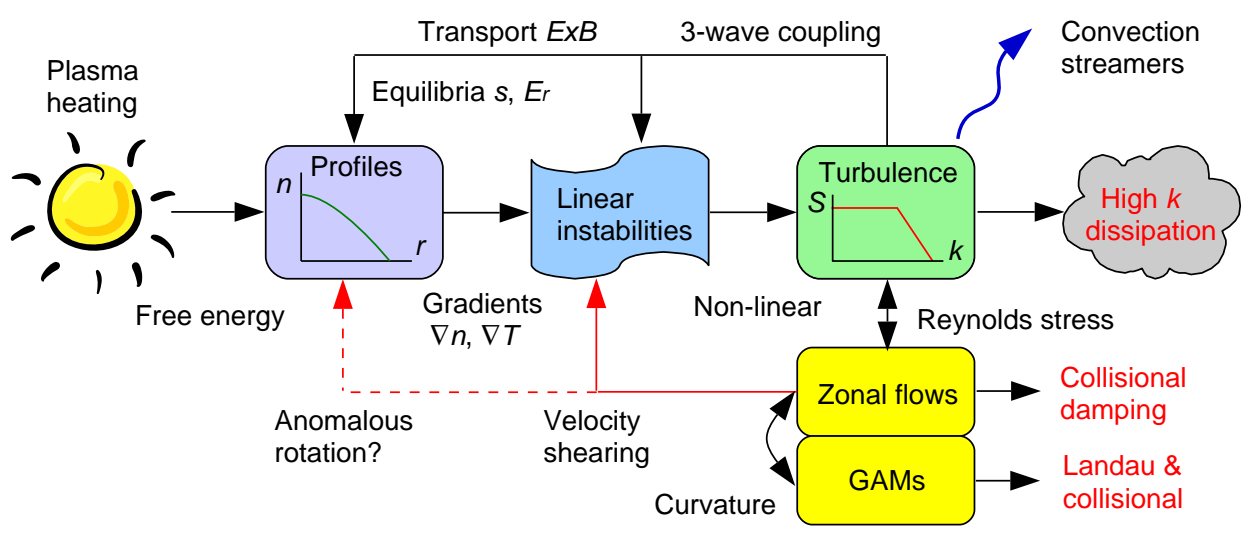

Figure 1. Schematic of energy flow and interaction of the various turbulence components in a magnetically confined plasma.

Connor's (1994) survey of anomalous transport theories [15]. There are several recent overview papers on turbulent transport modelling $[16,17,18]$.

\section{Turbulence energy flow}

Turbulence can be driven by many sources; stirring, as in astrophysical plasmas, velocity shearing (Kelvin-Helmholtz instabilities), fast particles (two-stream instabilities) etc. But in fusion confinement devices the main sources of free energy are the radial gradients in the plasma density and temperature. Theory predicts that tokamak and stellarator configurations are unstable to a variety of linear modes - drift-waves, ion and electron temperature gradient modes, trapped particle modes etc. [15]. These modes grow to saturation and lead, via non-linear three-wave processes, to fully developed broadband turbulence. For two-dimensional turbulence (approximately the case in magnetic confined plasmas) the mean squared turbulence vorticity as well as the kinetic energy are constrained - meaning, energy cascades both up and down the wavenumber $k$ range, creating the classic Kolmogorov/Kraichnan type power spectra [19]. At the high $k$ end the energy dissipates out into thermal processes via particle collisions and viscosity - see figure 1 - while at the low $k$ end the energy moves into large scale plasma flows. That is to say, the turbulent eddies or structures merge, forming ever larger ones, until eventually reaching scales comparable to the plasma dimensions. At this point the turbulence structures are essentially flows, comparable to the equilibrium plasma flows induced by external particle and momentum injection, and inherent plasma $E \times B$ rotation due to internal static radial and poloidal electric fields. These turbulence generated flows can affect both the background equilibrium conditions as well as the growth rates of the underlying instabilities. Turbulent Reynolds stress is another mechanism by which the turbulence can generate radially localized or zonal flows. The zonal flows can couple through the geodesic curvature of the confining magnetic field to create an eigenmode oscillation called the geodesic acoustic mode or GAM. Both zonal flows and GAMs are 

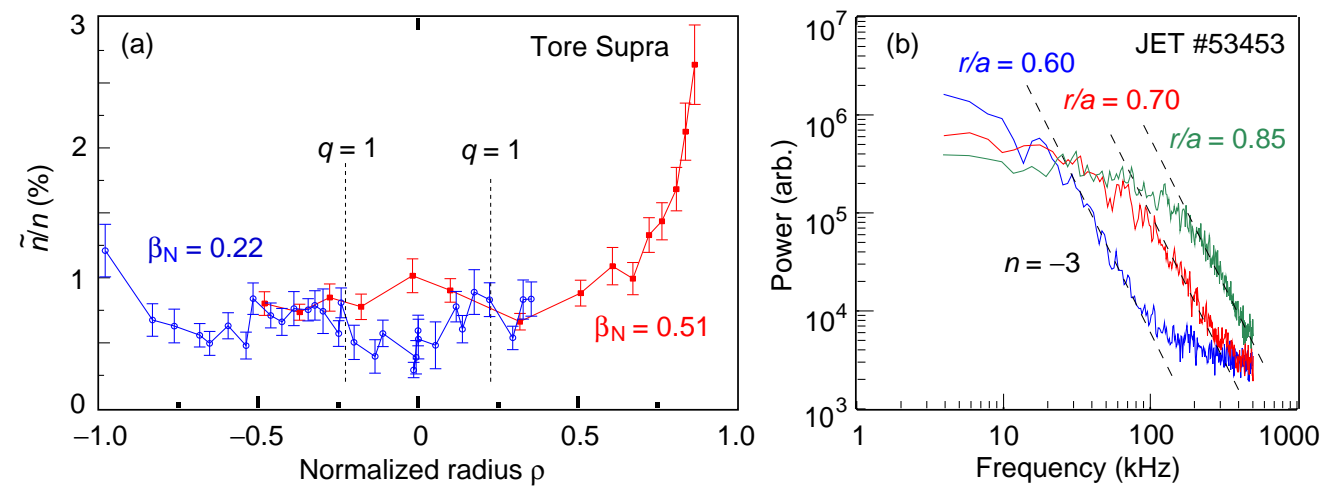

Figure 2. (a) Radial profile of density fluctuation level for two values of $\beta_{N}$ in ICRF heated Tore Supra discharges - reprinted from ref. [23]; and (b) Turbulence frequency spectra at various radii during Lower-Hybrid heated in JET - adapted from ref. [28].

parasitic on the turbulence and can also dissipate energy out through collisional and non-collisional Landau damping. The formation of static and oscillating flows form part of a closed loop which essentially regulates the turbulence through both vortex shearing (stretching, twisting and even breaking the large eddies back into smaller ones) and by providing an additional energy sink. There is some speculation that the zonal flows could also be the source of the anomalous rotation observed in many experiments [20].

\section{Turbulence features}

The universality of the turbulence features is often stated; statistically random fluctuations over a wide scale range (wavenumber) with typically a spectral dependence $S(k)=|n(k)|^{2} \propto k^{-\alpha}$ with $\alpha \sim 3.5 \pm 0.5$. The turbulence is anisotropic due to the fast parallel particle conduction along the confining magnetic field $B$; meaning fluctuations stretch out parallel to $B$ with $k_{\|}<k_{\perp} \sim k_{\mathrm{r}}$ - ie. the turbulence is roughly $2 \mathrm{D}$.

The turbulence can generate convective particle and thermal energy fluxes in both the electrons and ions $(j)$ : (particle) $\Gamma_{j}=\left\langle\tilde{n}_{j} \tilde{v}_{E \mathrm{r}}\right\rangle+n_{j}\left\langle\tilde{v}_{j \|} \tilde{B}_{\mathrm{r}}\right\rangle / B$ and (thermal) $Q_{j}=\frac{3}{2}\left\langle\tilde{p}_{j} \tilde{v}_{E \mathrm{r}}\right\rangle+\left\langle\tilde{q}_{j \|} \tilde{B}_{\mathrm{r}}\right\rangle / B+\frac{5}{2} p_{j}\left\langle\tilde{v}_{j \|} \tilde{B}_{\mathrm{r}}\right\rangle / B$, where the ensemble averages of the cross products mean the relative phases as well as the amplitudes of fluctuations in the density $\tilde{n}$, pressure $\tilde{p}$ (ie. temperature), parallel heat flux $\tilde{q}_{\|}$, velocities $\tilde{v}_{\|}$, electric field $\tilde{E}$ and magnetic field $\tilde{B}_{\mathrm{r}}$ are all important! Although measurements have been made of most of the individual parameters, it is still a challenge to measure all simultaneously; amplitudes and phases, with good spatial and temporal resolution - particularly in the plasma core. Typically RMS density fluctuation $\tilde{n}_{\mathrm{e}} / n_{\mathrm{e}}$ levels range from several to tens of percent close to the plasma boundary, dropping to a fraction of a percent in the core $[4,21,22]$. Figure 2(a) shows an example from microwave reflectometry on Tore Supra [23]. A strong correlation between increasing fluctuation level and decreasing confinement is often observed [24]. Ion and electron temperature fluctuations $\tilde{T}_{\mathrm{i}, \mathrm{e}} / T_{\mathrm{i}, \mathrm{e}}$ can be comparable to density $[25,26]$ and potential fluctuations. Magnetic fluctuations tend to be several orders of magnitude smaller than the density [4] - ie. the turbulent 
transport is predominantly electrostatic in nature.

The radial density fluctuation spectrum $S\left(k_{\mathrm{r}}\right)$ is broad and peaks around $k_{\mathrm{r}} \rho_{\mathrm{s}} \sim 0.1$ (where $\rho_{\mathrm{s}}$ is the ion gyro-radius at the sound speed) while the perpendicular (approx. poloidal) spectrum $S\left(k_{\perp}\right)$ peaks a little higher at $k_{\perp} \rho_{\mathrm{s}} \sim 0.2-0.4$ [21,22]. Corresponding correlation lengths, $L_{\mathrm{r}}$ and $L_{\perp}$, are similar and of the order of one $\mathrm{cm}$ or less in the edge, rising to several $\mathrm{cm}$ in the core $[21,22,27]$. The frequency spectrum, $S(\omega)=\left(v_{E_{\mathrm{r}} \times B}+v_{\mathrm{ph}}\right) \times S\left(k_{\perp}\right)$, scales directly with the $k$ spectrum and the plasma velocity (dominated by the $E_{\mathrm{r}} \times B$ rotation, since there is no net radial flow) plus the turbulence phase velocity $v_{\mathrm{ph}}$. Figure $2(\mathrm{~b})$ illustrates the frequency scaling in the JET tokamak where the spectra broaden with increasing poloidal spin-up towards the plasma edge but maintain a constant spectral index of $n=-3$ [28]. The dispersion relation appears linear for $k_{\perp} \lesssim 10 \mathrm{rad} / \mathrm{cm}[29,30]$, but the phase velocity may deviate at higher $k$ depending on the mode. The turbulence amplitude generally scales linearly with the temperature or density gradient, but often displays a threshold, ie. a critical gradient particularly for core electron type turbulence [31,32]. The debate is whether the scaling is with the gradient or the normalized gradient, e.g. $L_{T \mathrm{i}, \mathrm{e}}^{-1}=\nabla T_{\mathrm{i}, \mathrm{e}} / T_{\mathrm{i}, \mathrm{e}}$.

\section{Evidence for linear instabilities}

Magnetised plasmas are expected to be unstable to a variety of linear instabilities or modes. These include various branches of the classic electron drift-wave (EDW) driven by the electron pressure gradient [14], various forms of the ion temperature gradient mode (ITG) - toroidal and slab [17], the trapped electron mode (TEM) [15] and the electron temperature gradient mode (ETG) [33]. Experimentally, all turbulence appears to have a drift-wave-like nature, ie. it has a finite parallel wavenumber $k_{\|} \sim 1 / q R$ compared to a flute instability where $k_{\|} \rightarrow 0$. However, the individual modes should respond differently to the plasma conditions, for example TEM turbulence is expected to be strongly damped at high collisionality due to de-trapping of electrons. TEM is also believed to reduce density profile peaking, which impacts on the fusion performance and impurity retention in the core $[34,35]$. Thus, it is important to identify the dominant turbulence mechanism, not just for theory and code validation.

Most evidence for mode existence is somewhat indirect and comes from comparing global transport measurements with model predictions cf. [15, 5, 36, 34, 37, 38], but actual measurements of turbulence are less extensive. As numerical turbulence codes and synthetic diagnostics become more realistic, it is becoming possible to compare models directly with turbulence measurements, e.g. [39, 40, 41, 27, 43, 42, 26]. However, contrasting turbulence amplitudes or correlation lengths requires care as they are also subject gradient and shearing effects. An alternative turbulence property is the dispersion relation, or more simply the direction and magnitude of the phase velocity. Ion modes (ITG) will propagate in the ion diamagnetic drift direction and electron modes (EDW, TEM, ETG) in the opposite. In the laboratory frame the measured velocity is $u_{\perp}=v_{E_{r} \times B}+v_{\mathrm{ph}}$, the plasma plus phase velocity [30]. All turbulence experiences the 


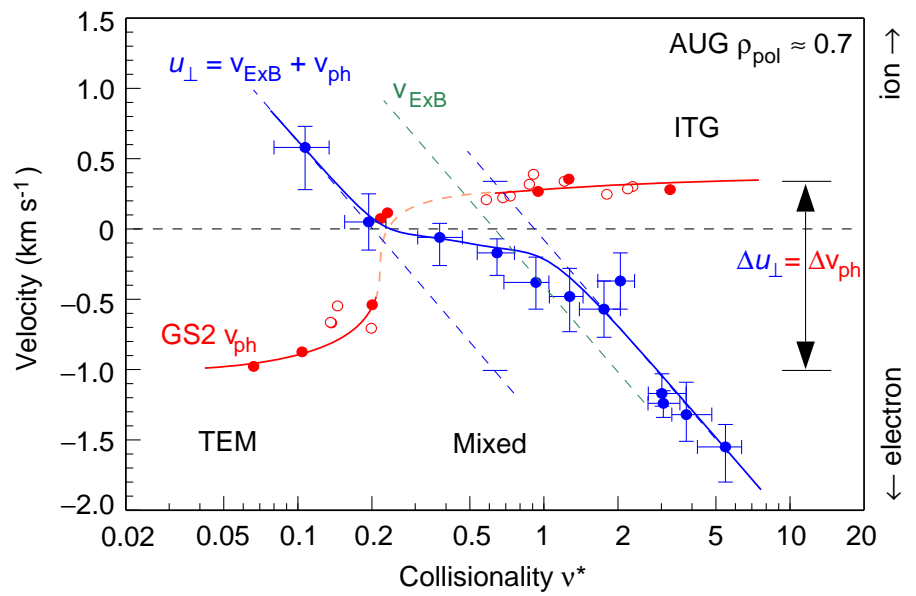

Figure 3. Measured turbulence velocity $u_{\perp}$ and gyrokinetic simulated phase velocity $v_{\text {ph }}$ vs collisionality in ohmic ASDEX Upgrade shots - adapted from ref. [46].

same $E_{\mathrm{r}} \times B$ velocity but the spectral peaks of ion and electron modes will be separated by their phase velocities - as illustrated by Brower's laser scattering measurements on the TEXT tokamak [29]. However, questions over spatial localization and diagnostic issues [44] left the issue of simultaneous mode existence unresolved. Turbulence simulations suggest simultaneous modes may exist, but not at the same $k$ [45].

A spatially localized and $k$ selective measurement from the ASDEX Upgrade tokamak core is shown in figure 3 where the turbulence $u_{\perp}$ from Doppler reflectometry is contrasted with computed linear mode phase velocities from the gyrokinetic code GS2 for a series of ohmic discharges with increasing collisionality $\nu^{*}$ [46]. The $u_{\perp}$ displays a background $\left(v_{E \times B}\right)$ logarithmic variation with $\nu^{*}$, but with a displacement. The step in velocity matches the magnitude, direction and location of the jump in the predicted phase velocity $\Delta u_{\perp}=\Delta v_{\mathrm{ph}}$ from TEM to ITG dominant regimes. Between the regimes $u_{\perp}$ takes a value which is a mixture of TEM and ITG velocities - there is no "peak splitting" ie. no simultaneous modes at the same probed $k_{\perp} \sim 10 \mathrm{rad} / \mathrm{cm}$.

\section{Electrostatic vs electromagnetic turbulence}

Is turbulent transport dictated by electrostatic (e.g. pressure/potential) or magnetic fluctuations? Theory indicates that at low plasma $\beta$ (ratio of plasma to magnetic pressure) electromagnetic (EM) effects are negligible [47]. Indeed, for normal tokamak and stellarator conditions the evidence appears overwhelmingly for electrostatic. However, for tight or low aspect ratio spherical tokamaks $\beta$ becomes significant, which may drive microtearing or kinetic ballooning modes in the core, or influence the electrostatic turbulence; hence, EM effects have become an issue again.

The experimental evidence has been periodically assessed: Wootton (1990) [4], Connor (1993) [2], Bickerton (1997) [47]; but are generally inconclusive. The behaviour is complex and the data incomplete. The first internal measurements of core magnetic 
fluctuations using a cross-polarization microwave scattering diagnostic on Tore Supra $[31,32]$ found $\tilde{B} / B$ increasing with the electron temperature gradient - which is highly suggestive. However, $\tilde{n} / n$ also rose in concert; plus $\tilde{B} / B$ is orders of magnitude smaller. The question, is $\tilde{B} / B$ due to $\tilde{n} / n$ or vice versa? More recent Tore Supra measurements using reflectometry found no change in the low confinement L-mode density turbulence level across the plasma when the normalized $\beta_{\mathrm{N}}$ was doubled from 0.23 to 0.5 consistent with the measured weak degradation of the global energy confinement time $\tau_{E} \propto \beta^{-0.2 \pm 0.2}$ (compared to the ITER L-mode prediction of $\beta^{-1.4}$ ) [23, 48] which leaves only a small role for $\tilde{B} / B$. In ASDEX Upgrade Kurzan [49] found the radial correlation length of edge density turbulence increasing with local $\beta$ for $\mathrm{L}$ and H-mode plasmas. The implication being that electromagnetic effects could be important even in L-mode.

Regarding the spherical tokamaks: NSTX operating with very high $\beta_{\mathrm{N}} \sim 5.7$ found no $\beta$ dependence - ie. no magnetic fluctuations - in H-mode conditions [50, 51]. Ion thermal transport fell towards neoclassical levels $\chi_{\mathrm{i}} \rightarrow \chi_{\mathrm{ineo}}$ but electron thermal transport $\chi_{\mathrm{e}}>\chi_{\mathrm{i}}$ and is attributed to high- $k$ turbulence measured with a tangential scattering diagnostic [50] - see below. The situation is similar on MAST; high $E \times B$ flow shear appears to suppress the ion transport with remaining transport in the electrons [52]. Unfortunately there are no turbulence measurements from MAST, however, numerical simulations reveal a variety of ETG, microtearing and streamer activity together with significant electromagnetic effects [53]. The picture thus remains inconclusive and in need of futher measurements.

\section{High $k$ and the search for ETG turbulence}

Many devices report a reduction in ion transport down to neoclassical levels when the confinement is optimized, e.g. with edge or internal transport barriers etc. However, the electron thermal transport remains anomalously high - this is generally attributed to unsuppressed ETG or high wavenumber TEM turbulence. Electron modes such as ETG and TEM are expected at shorter wavelengths since they scale with the electron Larmor radius $\rho_{\mathrm{e}}$ rather than the larger ion Larmor radius $\rho_{\mathrm{i}}$ associated with ion modes.

High $k$ measurements are particularly difficult to make due to the low fluctuation levels, but results from dedicated experiments are now appearing. For example on Tore Supra far-forward laser scattering [54, 55] and Doppler reflectometry [48] have been used to probe upto $k_{\perp} \sim 26 \mathrm{rad} / \mathrm{cm}$ or $k \rho_{\mathrm{i}} \sim 2.5$ in ohmic and ion heated ICRF plasmas. Gyrokinetic simulations suggest that ETG could be unstable, however, no evidence of high $k$ activity was observed in the measurement range. In fact, the turbulence amplitude drops above $k \rho_{\mathrm{i}} \sim 1.5$ with the spectral decay rising from $k^{-3}$ in the inertial range to $k^{-6}$, suggesting a break from $2 \mathrm{D}$ turbulence and additional damping effects. Nevertheless, the overall turbulence scaling seems close to gyro-Bohm predictions, $\chi \approx \rho^{*} \chi_{\mathrm{B}}=\left(\rho_{\mathrm{i}} / a\right) e T / B$ [54]. A similar behaviour was observed in the $S\left(k_{\mathrm{r}}\right)$ spectrum in the ohmic FT2 tokamak using an enhanced microwave backscattering diagnostic [56]. However, some indications of enhanced very high $k_{\mathrm{r}}$ fluctuations were 
noted when the electron temperature gradient was raised above the predicted ETG threshold of $L_{\mathrm{T}}<1.25 L_{\mathrm{n}}$. High $k$ measurements on the NSTX spherical tokamak have recently been reported using a radially scannable tangential microwave scattering measuring density fluctuations between $k_{\mathrm{r}}=2-25 \mathrm{rad} / \mathrm{cm}$. A broad turbulence reduction across the full $k_{\mathrm{r}}$ range was observed inside edge pedestal region during the L to H-mode transition [51, 50]. Recent measurements by Mazzucato [57] show an enhanced and broadened frequency spectrum for $k_{\perp} \rho_{\mathrm{S}} \sim 8-16$ in the NSTX core as the electron temperature gradient $\nabla T_{\mathrm{e}}$ steepend during HHFW electron heating.

This positive correlation of turbulence amplitude with $\nabla T_{\mathrm{e}}$ is corroborated by measurements on the DIII-D tokamak using a combination of far-infrared (FIR) forward scattering channels $\left(k_{\theta} \sim 1-15 \mathrm{rad} / \mathrm{cm}\right)$ and a dual channel microwave backward scattering diagnostic $\left(k_{\mathrm{r}} \sim 20 \& 39 \mathrm{rad} / \mathrm{cm}\right)$ with core electron cyclotron heating $(\mathrm{ECH})$ $[58,59]$. However, the enhanced turbulent activity appeared at all wavelengths, not just those in the expected ETG range $k \rho_{\mathrm{s}}>2$. Comparisons with the GKS linear gyrokinetic code showed qualitative agreement with core measurements, but not overall.

Also encouraging are preliminary results from the ASDEX Upgrade tokamak using Doppler reflectometry. Here, an enhancement in the high $k_{\perp} \rho_{\mathrm{s}}>1.5$ spectrum was observed with a simultaneous reduction at low $k_{\perp} \rho_{\mathrm{S}}<1$ across the plasma edge region during core deposited ECH heating [60]. However, there was no clear coincidence with $\nabla T_{\mathrm{e}}$ steepening. Unfortunately, it is still too early to pronounce that an unambiguous ETG signature has been found.

\section{Velocity and magnetic shear}

A velocity shear would normally be a source of instability - e.g. the Kelvin-Helmholtz instability - however, when combined with magnetic shear (varying magnetic field line helicity or pitch) which prevents the mode coupling across the velocity gradient, it can become a stabilizing factor. Fluid turbulence imaging, numerical simulations and edge tokamak turbulence imaging diagnostics, such as Beam Emission Spectroscopy and Gas Puff Imaging [61, 62], show that the velocity shear breaks-up or prevents the formation of large radial turbulent eddy structures - thus reducing direct convective transport.

Extensive experimental evidence - mostly from correlated turbulence reduction and improved confinement associated with transport barrier formation [63] - has established the radial gradient in the plasma $E_{\mathrm{r}} \times B$ (perpendicular) velocity (induced via momentum injection from neutral beam heating or plasma potential biasing) together with magnetic shear as the crucial factors. For example, Ritz (1990) [64] observed a drop in both density $\tilde{n}$ and potential $\tilde{\phi}$ fluctuations around a narrow radial edge region of $v_{E \times B}$ shear in the TEXT tokamak; since verified many times in the H-mode edge transport barrier. Similar behaviour is observed with internal transport barriers (ITB) where the broadband turbulence amplitude is temporally and spatially reduced but not fully suppressed, cf. [65]. Reductions in the turbulence radial correlation length structure size - have also beeb reported for edge [64, 66, 49,67] and core barriers [68]. 
See Gohil (2002) [69] for an overview of transport barriers and fluctuation behaviour.

Several theories have evolved to explain the sudden bifurcation in confinement at the H-mode transition [70]. These generally revolve around the $E_{\mathrm{r}}$ shear [71]. The suggestion of a threshold in shear is seen in the DIII-D tokamak where the edge $E_{\mathrm{r}}$ already begins to fall steadily prior to the rapid localized drop in $\tilde{n}$ [63]. However, the radial width of the $E_{\mathrm{r}}$ shear as well as its magnitude is also important. The evidence of the turbulence amplitude and/or turbulence cross-phase scaling with the $E_{\mathrm{r}}$ shear in both L-mode and into H-mode on the TEXTOR tokamak is also compelling [72]. Nevertheless, the picture is incomplete; there are questions about the $E_{\mathrm{r}}$ response after the fast turbulence drop; localized precursor spikes in the poloidal rotation have been observed in ITBs [73] and ETBs [74]; and often, after the initial drop the edge turbulence rises again [69] although the plasma remains in H-mode, indicating competition between the shear and turbulence drive as $\nabla p$ evolves. There is also the issue of the edge turbulence and $E_{\mathrm{r}}$ behaviour during ELM events [6].

Magnetic shear can also independently affect the turbulence growth rate. Experiments to decouple the effects of velocity and magnetic shear were performed on the JET tokamak using lower-hybrid heating to create an electron ITB - a central region of strong reversed shear with no momentum input, ie. no velocity shear. TEM-like turbulence was suppressed inside the reversed shear region, correlated with decreased electron transport $\chi_{\mathrm{e}}$ [28]. Similar reductions in core turbulence were seen in high shear conditions in Tore Supra [32].

To summarize, velocity shear appears effective in reducing the larger scale ion modes such as ITG, while magnetic shear (negative) acts more on the shorter scale electron modes such as TEM and ETG. Now, if mean $E \times B$ flow shear can affect the turbulence, what about oscillating flows?

\section{Zonal flows and GAMs}

The final part of the turbulence chain shown in figure 1 are the zonal flows (ZFs) and their oscillating counterpart, geodesic acoustic modes (GAMs) [75, 76, 77, 7]. Predicted by turbulence simulations (and overlooked in experiments for many years) they are now ubiquitously seen in almost all devices that have cared to look for them [78]. The recent explosion in reported measurements is a major development in the turbulence field, permitted in part by diagnostic advances that allow fluctuation measurements of the potential via heavy ion beam probes (HIBP) [79, 80], or in the flow field via BES [81] or Doppler reflectometry [82]. Advanced data processing techniques (such as bispectral analysis) of probe data provide convincing evidence of the non-linear drive of ZFs and GAMs and their back-reaction on the turbulence [83, 84], particularly the modulation of the turbulence amplitude [82, 7].

Both ZFs and GAMs are flows with an axisymmetric $(m=n=0)$ mode structure and a zonal ring nature, ie. finite $k_{\mathrm{r}} \neq 0$, cf. [78, 85]. Partly because of their quasistatic $(f \sim 0)$ and strong damping in the edge ZFs are more difficult to study. In 

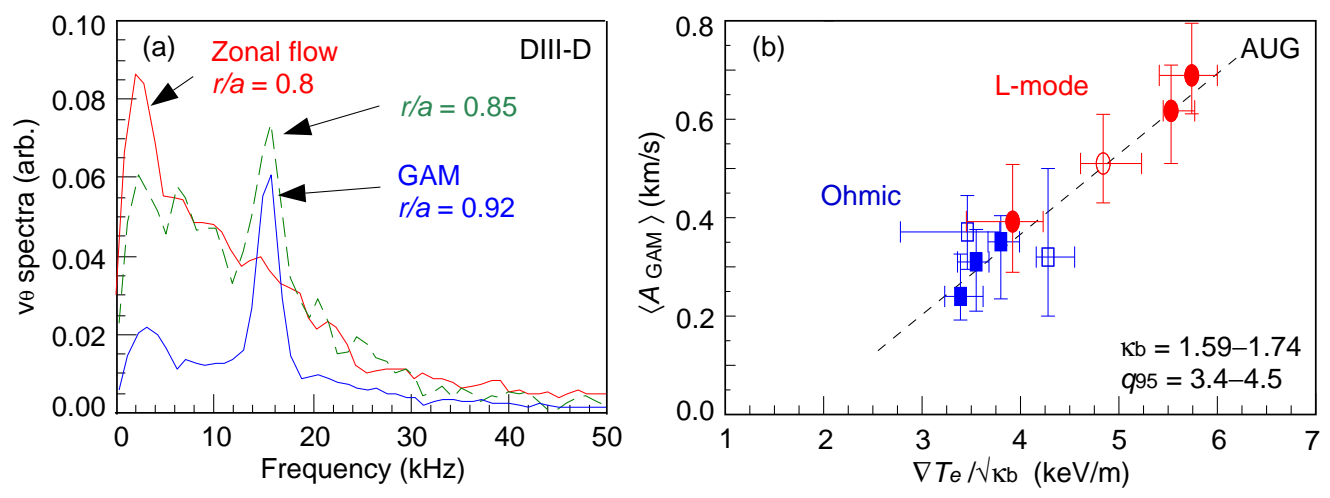

Figure 4. (a) Poloidal $v_{\theta}$ spectra from BES on DIII-D showing ZF to GAM transition with radius (Reprinted with permission from D Gupta et al, Phys. Rev. Lett. 97125002 (2006); Copyright (2006) by the American Physical Society); and (b) Mean GAM amplitude vs normalized electron temperature gradient from Doppler reflectometry on AUG - adapted from ref. [91].

contrast, GAMs are stronger in the edge and thus easier to access, plus they have an associated $m= \pm 1$ pressure perturbation. The issue of whether the ZF/GAM has a significant magnetic perturbation [86] and coupling to the Alfvén continuum is still evolving [87]. Figure 4(a) shows the poloidal velocity $v_{\theta}$ spectra from BES on DIII-D where a low frequency ZF in the core transitions to a GAM in the edge [88]. The GAM behaviour is complex. In circular limiter plasmas their frequency appears to scale in accordance with theory [89], while for shaped divertor tokamak plasmas it varies with elongation and $q_{95}[81,85]$. Indeed, theory now needs to catch-up with the experiment. The GAM amplitude also scales inversely with plasma elongation $\kappa_{b}$ - to be expected from reduced turbulence [90] - and directly with the normalized electron temperature gradient $\nabla T_{\mathrm{e}} / \sqrt{\kappa}_{b}$ as shown in figure 4 (b) from Doppler reflectometer data on ASDEX Upgrade (AUG), consistent with increasing turbulence drive and reduced confinement - upto the H-mode transition [91].

Although numerical simulations have demonstrated the effect of ZFs on reducing the turbulence radial correlation lengths down to measured values, i.e. radial shearing of the turbulence eddies [27], it will be a challenge to prove this conclusively by experiment

- since ZF/GAMs are not independent of the turbulence energy loop. The evidence for mean flow shear stabilization seems pretty solid, hence the role of core ZFs is believable. However, for GAMs to play a commensurate moderating role for the edge turbulence, and in particular to establish a role in turbulence stabilization and causality with the H-mode transition will require some effort.

\section{Radial streamers}

The formation of streamers - radially extended $\left(\sim 100 \rho_{\mathrm{e}}\right.$, i.e. several $\left.\mathrm{cm}\right)$ but poloidally narrow turbulent eddy structures have been predicted by numerical turbulence simulation codes $\mathrm{cf}$. [33, 16, 92] and references. They appear in simulations of ETG 
and TEM (electron) turbulence, where zonal flow activity is found to be weak, but are suppressed in ITG (ion) turbulence where the stronger poloidal shearing of ZF appears more readily to break-up the radial structures. Because of their large scale, streamers may cause significant convective cross-field transport in the core confinement region. Streamers are not to be confused with filaments and blob-like eruptions which have been extensively observed in the scrape-off-layer, and sometimes associated with ELM (edge localized modes) events [11, 12]. On the experimental side, the presence of streamers has been inferred from comparisons of GS2 gyro-kinetic predictions and measured electron heat fluxes $\chi_{\mathrm{e}}$ in MAST H-mode conditions $[52,53]$. They were also suggested to be the cause of non-MHD islands in early $T_{\mathrm{e}}$ measurements [2]. However, direct evidence for streamers is so far rather limited. Hammada et al [93] has recently presented observations of long range correlations in HIBP measurements in the JIPP TIIU tokamak interior. Large scale radially propagating events have also been observed in ECE measurements of $T_{\mathrm{e}}$ on the DIII-D tokamak [94] which are interpreted as avalanchelike phenomena. Until more conclusive measurements become available this will remain a topic of some debate.

\section{Final words}

By its nature this overview has had to be selective in the topics covered and the illustrative examples chosen. There are some glaring omissions; such as turbulence spreading - partly because there are no measurements [7], plus critical gradients and ZF induced shifts in turbulence thresholds. Also in the difficult to measure category is the topic of self-sustained turbulence generated by linearly stable modes [95]. The discussion of diagnostic developments - which underpin the measurements - is unfortunately rather curt, as the aim was to concentrate on the physics. The coverage is also in places rather superficial, particularly on the H-mode. Nevertheless, hopefully a flavour of the progress, the complexity and interrelatedness of measurements and theory, and some of the outstanding issues in the field can be seen.

\section{Acknowledgments}

C Angioni, B Scott and F Jenko are warmly acknowledged for many entertaining and fruitful discussions. R Sabot and D Gupta are thanked for permission to use figures.

\section{References}

[1] Hinton F L and Hazeltine R D 1976 Rev. Mod. Phys. 48239

[2] Connor J W 1993 Plasma Phys. Control. Fusion 35 B293

[3] Liewer P C 1985 Nucl. Fusion 25543

[4] Wootton A J, Carreras B A, Matsumoto H, McGuire K, Peebles W A, Ritz Ch P, Terry P W and Zweben S J 1990 Phys. Fluids B 22879

[5] Sheffield J 1994 Rev. Mod. Phys. 661015

[6] Hugill J 2000 Plasma Phys. Control. Fusion 42 R75 
[7] Burrell K H 2006 Plasma Phys. Control. Fusion 48 A347

[8] Bretz N 1997 Rev. Sci. Instrum. 682927

[9] Conway G D, Schirmer J, Klenge S, Suttrop W, Holzhauer E and ASDEX Upgrade Team 2004 Plasma Phys. Control. Fusion 46951

[10] Hidalgo C 1995 Plasma Phys. Control. Fusion 37 A53

[11] Carreras B A 2005 J. Nucl. Mater. 337-339 315

[12] Zweben S J, Boedo J A, Grulke O, Hidalgo C, LaBombard B, Maqueda R J, Scarin P and Terry J L 2007 Plasma Phys. Control. Fusion 49 S1

[13] Wagner F and Stroth U 1993 Plasma Phys. Control. Fusion 351321

[14] Horton W 1999 Rev. Mod. Phys. 71735

[15] Connor J W and Wilson H R 1994 Plasma Phys. Control. Fusion 36719

[16] Garbet X 2001 Plasma Phys. Control. Fusion 43 A251

[17] Garbet X et al 2004 Plasma Phys. Control. Fusion 46 B557

[18] Peeters A G, Angioni C and Tardini G 2006 C. R. Physique 7592

[19] Kraichnan R H 1967 Phys. Fluids 101417

[20] Rice J E et al 2004 Nucl. Fusion 44379

[21] Fonck R J et al 1993 Phys, Rev. Lett. 703736

[22] McKee G R et al 2001 Nucl. Fusion 411235

[23] Sabot R et al 2006 Plasma Phys. Control. Fusion 48 B421

[24] Durst R D et al 1993 Phys, Rev. Lett. 713135

[25] Evensen H T et al 1998 Nucl. Fusion 38237

[26] White A E et al 2008 Phys. Plasmas 15056116

[27] Rhodes T L, Leboeuf J-N, Sydora R D et al 2002 Phys. Plasmas 92141

[28] Conway G D et al 2002 Plasma Phys. Control. Fusion 441167

[29] Brower D L, Peebles W A, Kim S K, Luhmann Jr. N C, Tang W M and Phillips P E 1987 Phys. Rev. Lett. 5948

[30] Yu C X, Brower D L, Zhao S J et al 1992 Phys. Fluids B 4381

[31] Colas L, Zou X L, Paume M et al 1998 Nucl. Fusion 38903

[32] Hoang G T, Saoutic B, Guiziou L et al 1998 Nucl. Fusion 38117

[33] Jenko F, Dorland W, Kotschenreuther M and Rogers B N 2000 Phys. Plasmas 71904

[34] Angioni C et al 2005 Phys. Plasmas 12040701

[35] Weisen $\mathrm{H}$ et al 2005 Nucl. Fusion $45 \mathrm{~L} 1$

[36] Ernst D R et al 2004 Phys. Plasmas 112637

[37] Ryter F, Angioni C, Peeters A G et al 2005 Phys. Rev. Lett. 95085001

[38] Tanaka K et al 2007 Fusion Sci. Technol. 5197

[39] Rettig C L et al 2001 Phys. Plasmas 82232

[40] Conway G D, Kurzan B, Scott B, Holzhauer E and Kaufmann M 2002 Plasma Phys. Control. Fusion 44451

[41] Ross D W, Bravenec R, Dorland W et al 2002 Phys. Plasmas 9177

[42] Ernst D R et al 2006 Proc. 21st IAEA Fusion Energy Conf. (Chengdu) (Vienna: IAEA) IAEACN-149/TH/1-3, http://www-pub.iaea.org/MTCD/Meetings/fec2006pp.asp

[43] Vershkov V A et al 2005 Nucl. Fusion 45 S203

[44] Bretz N, Nazikian R, Bergin W, Diesso M, Felt J and McCarthy M 1990 Rev. Sci. Instrum. 61 3031

[45] Görler T and Jenko F 2008 Phys. Rev. Lett. 100185002

[46] Conway G D, Angioni C, Dux R et al 2006 Nucl. Fusion 46 S799

[47] Bickerton R J 1997 Plasma Phys. Control. Fusion 39339

[48] Hennequin P et al 2006 Proc. 21st IAEA Fusion Energy Conf. (Chengdu) (Vienna: IAEA) IAEACN-149/EX/P4-36 and http://www-pub.iaea.org/MTCD/Meetings/FEC2006/

[49] Kurzan B et al 2000 Plasma Phys. Control. Fusion 42237

[50] Kaye S M et al 2007 Nucl. Fusion 47449 
[51] Menard J E et al 2007 Nucl. Fusion 47 S645

[52] Lloyd B et al 2007 Nucl. Fusion 47 S658

[53] Roach C M et al 2005 Plasma Phys. Control. Fusion 47 B323

[54] Hennequin P, Sabot R, Honoré C et al 2004 Plasma Phys. Control. Fusion 46 B121

[55] Hennequin P 2006 C. R. Physique 7670

[56] Gusakov E Z, Gurchenko A D, Altukhov A B et al 2006 Plasma Phys. Control. Fusion 48 B443

[57] Mazzucato E and NSTX Group 2007 49th APS DPP meeting - Bull.Am.Phys.Soc. 52 CO3.0002

[58] Rhodes T L et al 2007 Plasma Phys. Control. Fusion 49 B183

[59] Rhodes T L et al 2007 Phys. Plasmas 14056117

[60] Tröster C 2008 Ph.D. Thesis, Ludwig Maximillian University, Munich

[61] McKee G R et al 2007 Plasma Fusion Res. 2 S1025

[62] Zweben S J et al 2004 Nucl. Fusion 44134

[63] Burrell K H 1997 Phys. Plasmas 41499

[64] Ritz Ch P, Lin H, Rhodes T L and Wootton A J 1990 Phys, Rev. Lett. 652543

[65] Conway G D et al 2001 Plasma Phys. Control. Fusion 431239

[66] Burrell K H et al 1992 Plasma Phys. Control. Fusion 341859

[67] Schirmer J et al 2007 Plasma Phys. Control. Fusion 491019

[68] Nazikian R et al 1998 Proc. 17th IAEA Fusion Energy Conf. (Yokohama) (Vienna: IAEA) IAEA$\mathrm{CN}-69 / \mathrm{PDP}-03$

[69] Gohil P 2002 Plasma Phys. Control. Fusion 44 A37

[70] Connor J W and Wilson H R 2000 Plasma Phys. Control. Fusion 42 R1

[71] Terry P W 2000 Rev. Mod. Phys. 72109

[72] Boedo J A, et al 2002 Nucl. Fusion 42117

[73] Bell R E et al 1998 Plasma Phys. Control. Fusion 40609

[74] Ido T et al 2002 Phys, Rev. Lett. 88055006

[75] Waltz R E, Kerbel G D and Milovich J 1994 Phys. Plasmas 12229

[76] Diamond P H, Itoh S-I, Itoh K and Hahm T S 2005 Plasma Phys. Control. Fusion 47 R35

[77] Itoh K et al 2006 Phys. Plasmas 13055502

[78] Fujisawa A et al 2007 Nucl. Fusion 47 S718

[79] Ido T et al 2006 Plasma Phys. Control. Fusion 48 S41

[80] Melnikov A V et al 2006 Plasma Phys. Control. Fusion 48 S87

[81] McKee G R, Gupta D K, Fonck R J, Schlossberg D J, Shafer M W, and Gohil P 2006 Plasma Phys. Control. Fusion 48 S123

[82] Conway G D et al 2005 Plasma Phys. Control. Fusion 471165

[83] Nagashima Y et al 2006 Plasma Phys. Control. Fusion 48 S1

[84] Lan T et al 2008 Plasma Phys. Control. Fusion 50045002

[85] Conway G D, Tröster C, Scott B, Hallatschek K and ASDEX Upgrade Team 2008 Plasma Phys. Control. Fusion 50055009

[86] Fujisawa A et al 2008 Phys. Plasmas 15055906

[87] Smolyakov A I, Nguyen C and Garbet X 2008 Private communication: Kinetic theory of electromagnetic geodesic acoustic modes - To be published

[88] Gupta D K, Fonck R J, McKee G R, Schlossberg D J and Shafer M W 2006 Phys. Rev. Lett. 97 125002

[89] Winsor N, Johnson J L and Dawson J M 1968 Phys. Fluids 112448

[90] Kendl A and Scott B D 2006 Phys. Plasmas 13012504

[91] Conway G D and ASDEX Upgrade Team 2008 Plasma Phys. Control. Fusion 50085005

[92] Kendl A 2003 Phys. Plasmas 10317

[93] Hamada Y, Watari T, Nishizawa A et al 2006 Phys. Rev. Lett. 96115003

[94] Politzer P A et al 2002 Phys. Plasmas 91962

[95] Scott B D 1990 Phys. Rev. Lett. 653289 